\title{
SCATTERING PROPERTIES OF COMETARY GRAINS
}

\author{
D. B. Vaidya
}

Gujarat College, Ahmedabad-380006, India

\author{
J. N. Desai
}

PRL, Ahmedabad-380009, India

\begin{abstract}
Halley probes (Giotto, Vega) have shown that cometary grains can be extremely dark and might have fluffy structures. These probes have also detected submicron sized particles in the coma of the comet. In view of these observations and to interpret the observed data on several other comets we calculate the angular distribution of the scattered intensity, polarization and albedo for porous and compact particles. For these calculations, we apply discrete dipole approximation (DDA) first to the spheroidal particles, assumed to be made of small scattering elements (dipoles). Then we reduce the number of dipoles systematically to model the porous grains. We study the phase function, linear polarization, and albedo as a runction of grain size and packing density.
\end{abstract}

\section{Introduction}

The GIOTTO and VEGA missions to comet Halley measured the mass distribution of comet dust and found a population of very small submicron size grains, with mass $\mathrm{m}<10^{-15} \mathrm{~g}$. (McDonnell et al., 1987; Mazets et al., 1987). The geometric albedo, Ap of the grains from the ground based observations was found to be quite low for comet Halley (0.03 - 0.04, Tokunaga et al., 1986; Lamy et al., 1987). This was consistent with the GIOTTO and VEGA observations of dark nucleus of comet Halley. The density of the nucleus of Halley was also found to be quite low, around $0.1-0.5 \mathrm{~g} / \mathrm{cm}^{3}$ (Whipple 1987). This suggests that comet grains are porous and fluffy as had been predicted earlier (Greenberg \& Gustafson 1981). In this paper first we show how the porous grains are generated. Then an attempt is made to show the effect of porosity on the scattering properties (i.e., phase function, polarization and albedo) of the particles.

\section{Discrete Dipole Approximation (DDA) and Porous Grain Models}

The discrete dipole approximation (DDA) is a flexible and very useful technique to study the scattering by targets of arbitrary shape. It was first used by Purcell \& Pennypacker (1973) and later developed by Draine (1988). The DDA replaces the solid particle by an array of $\mathrm{N}$ point dipoles, with the spacing between the dipoles small compared with the wavelength. The dipoles are assumed to occupy 
positions on a cubic lattice. We use the DDA program, ddscat.4b, developed by Draine \& Flatau (1994a) to generate the porous grains. There are two criteria for the validity of DDA; (i) mkd $<1$, where $\mathrm{m}$ is the complex index of refraction, $\mathrm{k}=2 \pi / \lambda, \lambda$ is the wavelength, and $\mathrm{d}$ is the dipole lattice spacing (ii) $\mathrm{d}$ must be small enough ( $\mathrm{N}$ must be large enough) to describe the target shape satisfactorily (Draine \& Flatau,1994b). The program ddscat.4b contains routines to generate arrays representing targets of various geometries. Initially we assumed the number of dipoles $N_{x}=25, N_{y}=N_{z}=20$ along the $\mathrm{x}, \mathrm{y}$, respectively z axis of the spheroidal target grain. This would result in the total number of dipoles $\mathrm{N}=5252$ in the solid grain. Then we reduce $N_{x}, N_{y}$ and $N_{z}$ systematically to generate the porous grains (viz. 20,15,15;15,10,10;10,5,5 and so on). Wolff et al. (1993) also used this program to model porous and fluffy particles.

\section{Results and Discussion}

Using DDA we have calculated the phase function and polarization for the porous grains with the refractive index $m=1.70,0.1$ (taken to be representative of astronomical silicate in the optical regime).

Figure 1 shows the phase function (Draine \& Flatau, 1994a; Draine, 1988; eq. 3.12) for three values of $\mathrm{N}$, i.e., (1) $\mathrm{N}=5252$, (2) $\mathrm{N}=808$, (3) $\mathrm{N}=80$. The crosses show the values calculated using Mie theory for $\mathrm{m}=1.70,0.1$ and grain size of radius $0.15 \mu \mathrm{m}$ at wavelegth $0.6280 \mu \mathrm{m}$. It is seen from these results that the curve 1 with $\mathrm{N}=5252$ is close to the Mie curve whereas curve 2 with $\mathrm{N}=808$ and curve 3 with $\mathrm{N}=80$ deviate from the Mie curve.

Figure 2 shows the polarization versus scattering angle for (1) $\mathrm{N}=5252$, (2) $\mathrm{N}=808$ and (3) $\mathrm{N}=80$ for $\mathrm{m}=1.70,0.1$. These curves show that as the porosity increases the angle of maximum polarization shifts towards 90 degree. The crosses show the values calculated using Mie theory for $\mathrm{m}=1.70,0.1$ and grain radius of $0.15 \mu \mathrm{m}$. Earlier using microwave laboratory results Giese et al. (1978) and Greenberg \& Gustafson (1981) had shown large low density fluffy particles to produce large polarization peaking at 90 degree. Lamy \& Perrin (1991) have shown the effect of porosity on both the phase function and the polarization. We have also calculated the single scattering albedo, $\mathrm{w}=\mathrm{Q}_{s c a} / \mathrm{Q}_{\text {ext }}$; geometric albedo, $\operatorname{Ap}(180)$, and asymmetry factor $\mathrm{g}$ as a function of grain size for both porous $(\mathrm{N}=2728, \mathrm{~N}=1184, \mathrm{~N}=224)$ as well as compact particles at $0.6280 \mu \mathrm{m}$ wavelength. The results for $\mathrm{N}=1184$ are presented in Table 1 . These results indicate that in general the geometric albedo $A p(180)$ is lower for the porous particles. Since these scattering parameters are studied as a function of grs in size with the smallest size being $0.01 \mu \mathrm{m}$ the initial value of $\mathrm{N}$ is kept lo: satisfies the criteria for DDA (Draine \& Flatau,1994b).

\section{Conclusions}

Using discrete dipole approximation (DDA) we have studied the scatterir erties of the porous particles. Our results show that the phase function : larization for porous particles deviate considerably from results obtained solid particles. Results on the geometric albedo seem to indicate that it is $\mathrm{i}$ for the porous particles. In this study we have considered single sized particles 
Fig. 1

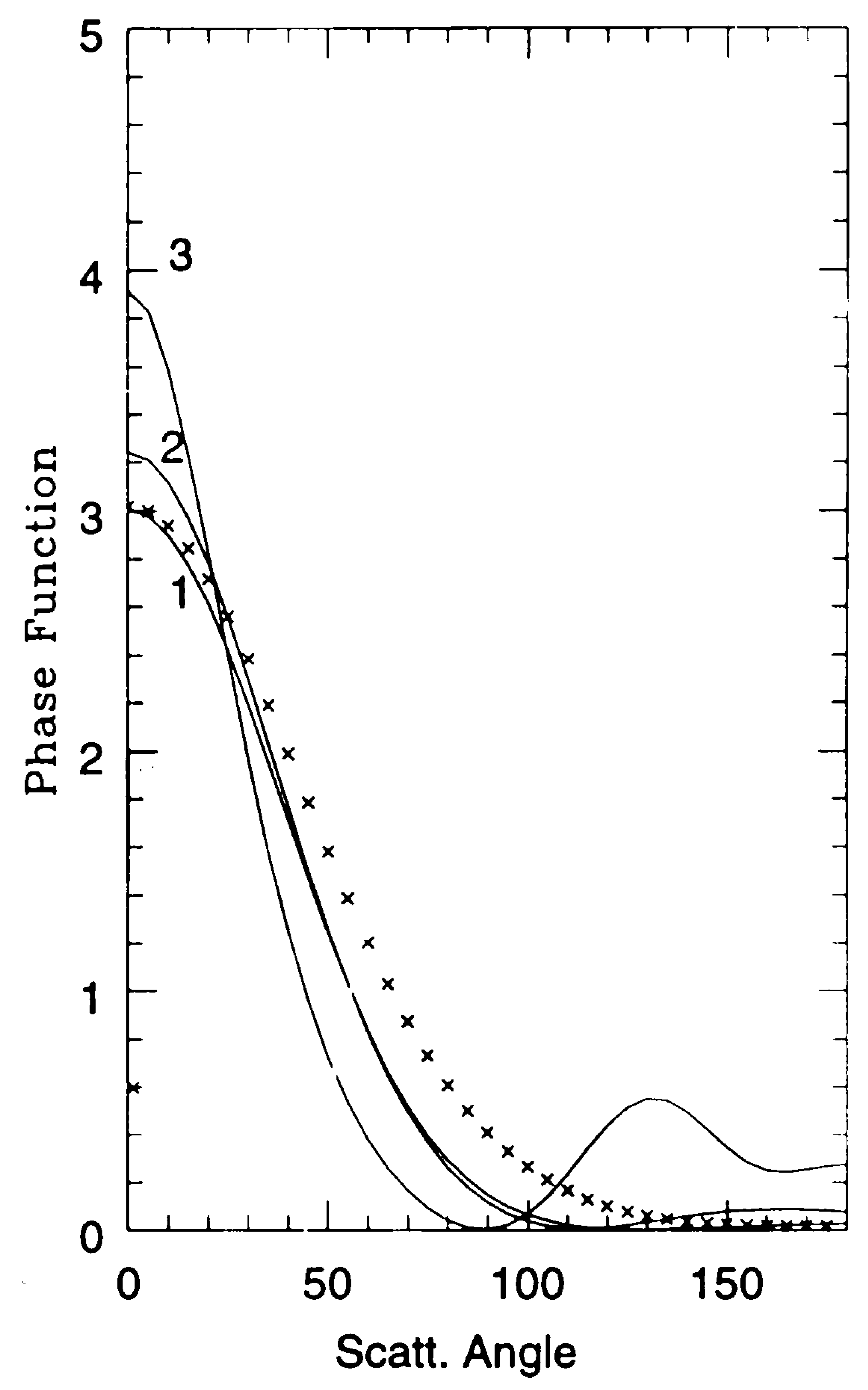

Fig.2

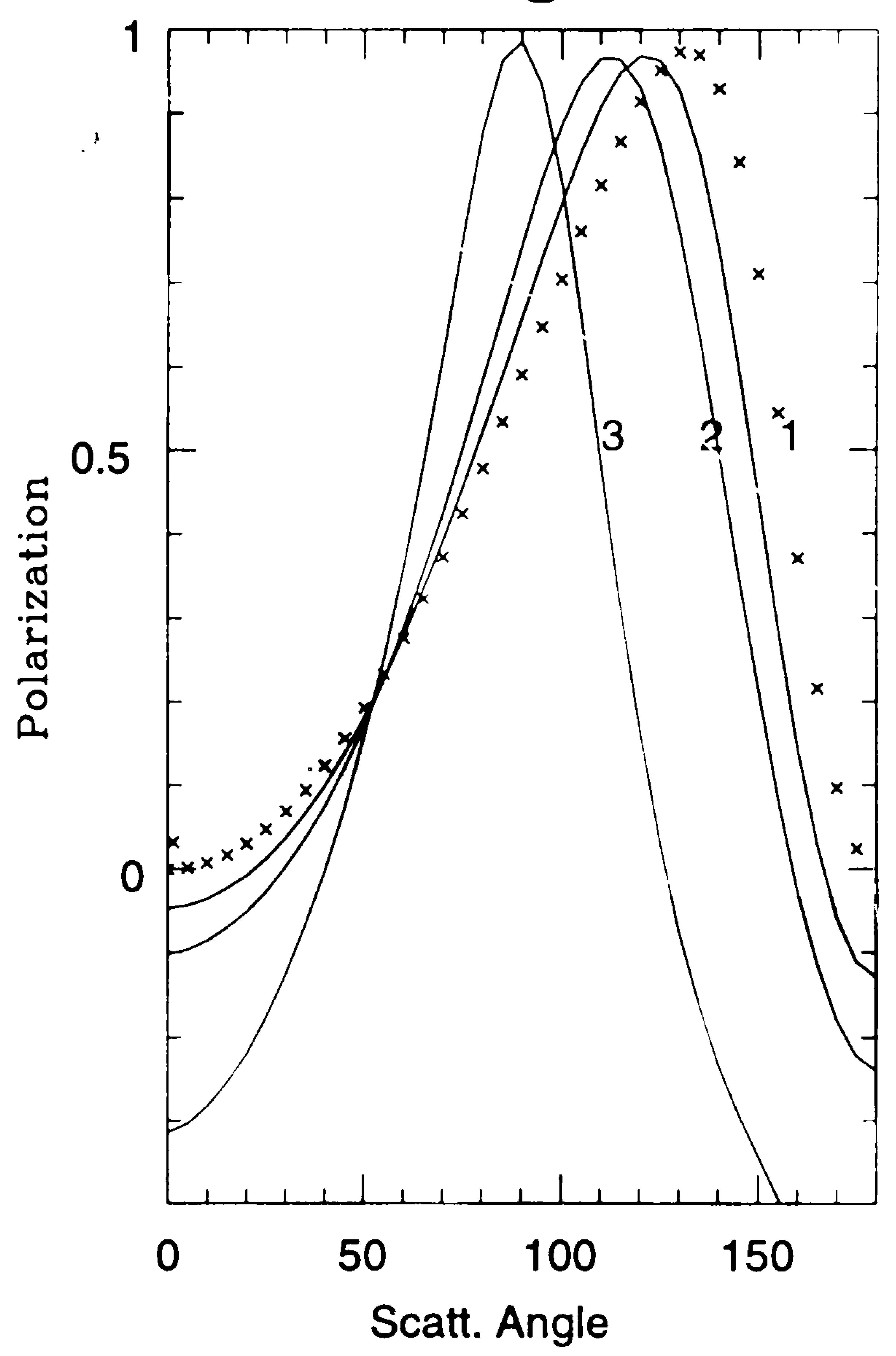

rure 1. Phase function for (1) $\mathrm{N}=5252$, (2) $\mathrm{N}=808$, (3) $\mathrm{N}=80$, $\mathrm{g} \mathrm{m}=1.7,0.1$. Crosses show the values calculated using Mie theory $n=1.70,0.1$ and grain size of $0.15 \mu \mathrm{m}$. Figure 2. Polarization us scattering angle for the same particle parameters as in Figure 1. 
and only one refractive index. However, in order to interpret the observed data on cometary dust we should use a size distribution in the size range of $.001 \mu \mathrm{m}$ to $1.0 \mu \mathrm{m}$ (and larger). This would require considerably large $\mathrm{N}(>10000)$ to satisfy the validity criteria of DDA. We plan to pursue this study using a super computer. We have also calculated the scattering parameters of the porous particles with $\mathrm{m}=1.33,0.0 ; \mathrm{m}=1.33,0.1 ; \mathrm{m}=1.70,0.0$. The analysis of these results is in progress (Vaidya \& Desai, 1996). As shown earlier the laboratory study would also help in interpreting the observed data on cometary dust.

\begin{tabular}{ccccccc}
$\begin{array}{c}\text { grain } \\
\text { radius } \\
\mu \mathrm{m}\end{array}$ & $\mathrm{w}$ & $\begin{array}{c}\text { Ap } \\
(180)\end{array}$ & $\mathrm{g}$ & $\mathrm{w}$ & $\mathrm{Ap}$ & $\mathrm{g}$ \\
& $(1184)$ & $(1184)$ & $(1184)$ & $(\mathrm{MIE})$ & $(\mathrm{MIE})$ & $(\mathrm{MIE})$ \\
\hline 0.01 & 0.002 & 0.000 & 0.002 & 0.002 & 0.0 & 0.002 \\
0.02 & 0.017 & 0.000 & 0.008 & 0.018 & 0.0 & 0.008 \\
0.03 & 0.051 & 0.000 & 0.021 & 0.057 & 0.0 & 0.019 \\
0.04 & 0.117 & 0.000 & 0.038 & 0.122 & 0.0 & 0.034 \\
0.05 & 0.207 & 0.000 & 0.060 & 0.207 & 0.000 & 0.053 \\
0.06 & 0.297 & 0.000 & 0.087 & 0.300 & 0.000 & 0.077 \\
0.07 & 0.388 & 0.001 & 0.119 & 0.389 & 0.001 & 0.106 \\
0.08 & 0.468 & 0.004 & 0.156 & 0.468 & 0.009 & 0.140 \\
0.09 & 0.535 & 0.011 & 0.200 & 0.534 & 0.024 & 0.180 \\
0.10 & 0.588 & 0.026 & 0.251 & 0.585 & 0.033 & 0.229 \\
0.11 & 0.626 & 0.043 & 0.311 & 0.623 & 0.063 & 0.289 \\
0.12 & 0.653 & 0.072 & 0.380 & 0.648 & 0.095 & 0.359 \\
0.13 & 0.671 & 0.085 & 0.450 & 0.665 & 0.100 & 0.437 \\
0.14 & 0.683 & 0.076 & 0.522 & 0.677 & 0.082 & 0.512 \\
0.15 & 0.695 & 0.046 & 0.573 & 0.690 & 0.027 & 0.568
\end{tabular}

Acknowledgments. Prof. B.T. Draine kindly provided the ddscat.4b code.

\section{References}

Draine B. T., 1988, ApJ, 333, 848

Draine B. T. \& Flatau P. J., 1994a, User Notes for ddscat.4b

Draine B. T. \& Flatau P. J., 1994b, J. Opt. Soc. Am. A, 11, 1491

Greenberg J. M. \& Gustafson B. Å. S., 1981, A\&A, 93, 35

Giese R. H., Weiss K., Zerull R. H. \& Ono T., 1978, A\&A, 65, 265

Lamy P L., Grün E. \& Perrin J. M., 1987, A\&A, 187, 767

Lamy P. L. \& Perrin J. M., 1991, in Origin and Evoltion of Interplanetary Dust, (eds. A.C.Levasseur-Regourd, Hasegawa H.), Kluwer, Dordrecht, 163

Mazets E. P. et al., 1987, A\&A, 187, 699

McDonnell J. A. M. et al., 1987, A\&A, 187, 719

Purcell E. M. \& Pennypacker C. R., 1973, ApJ, 186, 705

Tokunaga A. T et al., 1986, AJ, 92, 1183

Vaidya D. B. \& Desai J. N., 1996, in preparation

Whipple F. L., 1987, A\&A, 187, 852

Wolff M. J., Clayton G. C., Martin P. G. \& Sculte-Ladback R. E., 1993, Wisconsin Astrophysics Preprint No 490 